\title{
The faint 2005 hard state outburst of Aquila X-1 seen by INTEGRAL and RXTE (Research Note)
}

\author{
J. Rodriguez ${ }^{1}$, S. E. Shaw ${ }^{2,3}$, and S. Corbel ${ }^{1}$ \\ 1 AIM, Unité Mixte de Recherche CEA, CNRS, Université Paris VII - UMR 7158, CEA Saclay, Service d'Astrophysique, \\ 91191 Gif-sur-Yvette, France \\ e-mail: rodrigue@discovery.saclay.cea.fr \\ 2 School of Physics and Astronomy, University of Southampton, SO 17 1BJ, UK \\ 3 INTEGRAL Science Data Centre, 16 Chemin d'Ecogia, 1290 Versoix, Switzerland
}

Received 25 October 2005 / Accepted 7 February 2006

ABSTRACT

\begin{abstract}
We report on the spectral analysis of RXTE and INTEGRAL data of the 2005 April outburst of the transient Atoll source Aql X-1. Although this outburst is one of the faintest ever detected for this source in the soft X-rays (RXTE/ASM), one of our INTEGRAL observations, taken close to the soft X-ray peak, shows that the source flux was quite high, with a $20-200 \mathrm{keV}$ flux of $2.05 \times$ $10^{-9} \mathrm{erg} \mathrm{cm}^{-2} \mathrm{~s}^{-1}$. On this occasion we detect the source up to $150 \mathrm{keV}$ for the first time. We compare and discuss the similarity of the source behavior with that of black hole transients especially XTE J1550-564.
\end{abstract}

Key words. accretion, accretion disks - stars: individual: Aquila X-1 - stars: individual: XTE J1550-564 - stars: neutron $\mathrm{X}$-rays: binaries - gamma rays: observations

\section{Introduction}

Aql X-1 is a transient X-ray binary which undergoes outbursts about once per year. It is a Low Mass X-ray binary composed of a Roche-lobe filling late type $\mathrm{K}$ star orbiting a weakly magnetized neutron star at a distance 4.5-6 kpc (Chevalier et al. 1999; Rutledge et al. 2001). Along its outbursts it has been observed to transit through several spectral states, either characterized by a hard power law tail, and or the presence of a soft X-ray component usually modeled by an optically thick accretion disk (e.g. Maitra \& Bailyn 2004). The short recurrence time around 1 year and its evolution along the outbursts makes it an ideal target to study the accretion processes around compact objects.

Apart from very bright outbursts, Aql X-1 is sometimes seen to undergo "low luminosity" (in the soft X-rays) hard state outbursts (a.k.a mini-outburst). Although these were already reported with BATSE (Harmon et al. 1996), it is remarkable that the two last outbursts were of this type (e.g. Rodriguez et al. 2004; Maitra \& Bailyn 2005b, for the 2004 outburst). On April 1st 2005 the source was detected during an INTEGRAL Galactic Plane Scan (Grebenev et al. 2005). This detection followed the apparent reactivation of the source in optical (Maitra \& Bailyn 2005a), suggesting a new outburst was beginning. The source was again in the field of view of our INTEGRAL monitoring campaign of GRS 1915+105 (PI Rodriguez) 10 days and $~ 40$ days later. Very recently, Rodriguez \& Shaw (2005) reported renewed activity of Aql X-1 in the hard X-rays with INTEGRAL in the end of November 2005.

We report here the spectral analysis of the INTEGRAL and $R X T E$ observations. We start by giving the data reduction procedures in the next section, and present the results of our

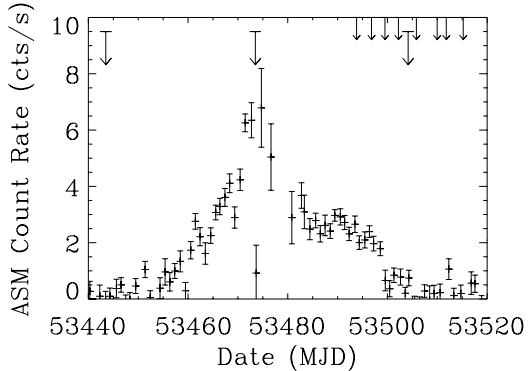

Fig. 1. 1.2-12 keV RXTE/ASM light curve of Aql X-1 during the 2005 outburst. The small arrows indicate the moment of RXTE dedicated pointings (PCA+HEXTE), and the long ones the dates of our INTEGRAL observations.

observation in Sect. 3. We briefly discuss our findings in the last part of the paper.

\section{Observations and data reduction}

Figure 1 shows the RXTE/ASM light curve during the 2005 outburst. The individual INTEGRAL pointings are indicated. The journal of the observations is reported in Table 1.

\subsection{INTEGRAL data reduction}

The INTEGRAL observations are part of a monitoring campaign on the microquasar GRS $1915+105$. Given that the field of views (hereafter FOV) of the main instruments on-board INTEGRAL are rather large (e.g. $29^{\circ} \times 29^{\circ}$ down to 0 response for IBIS/ISGRI see Lebrun et al. 2003), Aql X-1 is always in the 
Table 1. Journal of the INTEGRAL and RXTE observations presented in this paper. * Effective exposure of the Aql X-1 spectra, obtained after correction for gti and instrumental deadtime.

\begin{tabular}{cccc}
\hline \hline \multicolumn{4}{c}{ INTEGRAL } \\
\hline Rev. \# & Start & Stop & Effective exposure* \\
& MJD & $\left(\times 10^{3} \mathrm{~s}\right)$ \\
\hline 295 & 53442.95 & 53444.15 & Not detected \\
305 & 53472.87 & 53474.14 & 77 \\
315 & 53503.54 & 53504.85 & 51 \\
\hline \multicolumn{5}{c}{} & RXTE & \\
\hline Obs. \# & ObsId & Start & PCA Good Time \\
& & MJD & $(\mathrm{s})$ \\
\hline 1 & $91414-01-01-00$ & 53493.77 & 3216 \\
2 & $91414-01-02-00$ & 53496.79 & 2464 \\
3 & $91414-01-02-01$ & 53499.53 & 3200 \\
4 & $91414-01-02-02$ & 53502.24 & 2208 \\
5 & $91414-01-03-00$ & 53505.83 & 1728 \\
6 & $91414-01-03-01$ & 53509.97 & 1936 \\
7 & $91414-01-04-00$ & 53511.80 & 256 \\
8 & $91414-01-04-01$ & 53515.17 & 752 \\
\hline
\end{tabular}

field of view of both IBIS and SPI during our campaign. This is, however, not true for the JEM-X monitors that have smaller FOV, as a consequence, Aql X-1 is always outside of the JEM-X FOV.

The IBIS/ISGRI data were reduced with the version 5.0 of the Off-line Scientific Analysis (OSA) software. The sequence of procedures we performed are similar to those reported in e.g. Rodriguez et al. (2006), with production of images in the $20-40$ and $40-80 \mathrm{keV}$ ranges, leaving the software free to find the most significant sources in the field, and extraction of spectral products for the most active sources in the field. The OSA 5 response matrices were used for spectral analysis. We extracted spectra from the SPI instrument, but due to a lower sensitivity than ISGRI below $200 \mathrm{keV}$, these does not provide additional information, and are therefore not included here.

\subsection{RXTE data reduction}

The RXTE data were reduced with the version 6.0.2 of the HEASOFT software. For the Proportional Counter Array (PCA), we extracted spectral products from the top layer of Proportional Counter Units (PCU) \#0 and \# 2 that were the only ones always turned on throughout the outburst. We restrained the spectral analysis of the High Energy Timing Experiment (HEXTE) to Cluster A only. For both instruments the data reduction was done in a similar manner as in Rodriguez et al. (2003), using the faint background maps to estimate the PCA background, and filtering out time with rate of high electron background in PCU 2 (keeping time with electron $2<0.1$ ). We then applied $0.6 \%$ systematics to all PCA channels. The resultant spectra were fitted simultaneously in XSPEC v11.3.2 in the 3-25 keV (PCA) and $18-180 \mathrm{keV}$ (HEXTE) energy ranges. A normalization constant was included to account for uncertainty in the instrumental cross-calibration. Freezing that of the PCA data to 1 the value of the constant for the HEXTE spectra was always found between 0.95 and 1.1 .

\section{Results}

\subsection{INTEGRAL observations}

Aql X-1 is not detected by INTEGRAL/ISGRI during our first observation, with $3 \sigma$ upper limits of $0.37 \mathrm{cts} / \mathrm{s}$ $\left(\sim 3.3 \mathrm{mCrab}^{1} \approx 2.7 \times 10^{-11} \mathrm{erg} \mathrm{cm}^{-2} \mathrm{~s}^{-1}\right)$ and $0.33 \mathrm{cts} / \mathrm{s}$ $\left(\sim 5.5 \mathrm{mCrab} \approx 3.9 \times 10^{-11} \mathrm{erg} \mathrm{cm}^{-2} \mathrm{~s}^{-1}\right)$ in the $20-40 \mathrm{keV}$ and $40-80 \mathrm{keV}$ energy ranges. Our first detection occurs on MJD 53472, with fluxes of $16.13 \mathrm{cts} / \mathrm{s}(\sim 150 \mathrm{mCrab} \approx 1.19 \times$

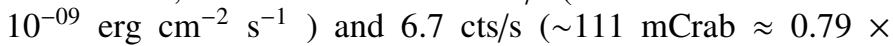
$\left.10^{-09} \mathrm{erg} \mathrm{cm}^{-2} \mathrm{~s}^{-1}\right)$ in the same ranges. During our last observation, the flux of Aql X-1 has significantly decreased to a level of $0.8 \mathrm{cts} / \mathrm{s}\left(7 \mathrm{mCrab} \approx 5.8 \times 10^{-11} \mathrm{erg} \mathrm{cm}^{-2} \mathrm{~s}^{-1}\right)$ in the $20-40 \mathrm{keV}$ range, while it is not detected at higher energy with a $3 \sigma$ upper limit of $0.46 \mathrm{cts} / \mathrm{s}\left(\sim 7.6 \mathrm{mCrab} \approx 5.4 \times 10^{-11} \mathrm{erg} \mathrm{cm}^{-2} \mathrm{~s}^{-1}\right)$ in the $40-80 \mathrm{keV}$ range. The best fit spectral parameters are shown in Table 2. The ISGRI spectrum of Rev 305, shown in Fig. 2, is well described between 20 and $200 \mathrm{keV}$ by a power law with a high energy cut-off. One month later, in Rev 315, the source's hard and soft X-ray flux has decreased dramatically and the spectral parameters are poorly constrained. A simultaneous fit of the ISGRI Rev 315 and PCA+HEXTE Obs 5 spectra is discussed in Sect. 3.3.

\subsection{RXTE observations}

The PCA+HEXTE spectra were first fitted with a simple model of an absorbed power law. We first left $N_{\mathrm{H}}$ free to vary in the fits, but the latter always tended to values close to 0 . Given the $3 \mathrm{keV}$ lower boundary of the PCA bandpass, we fixed $N_{\mathrm{H}}$ to the Galactic value along the line of sight $\left(N_{\mathrm{H}}=0.34 \times\right.$ $10^{22} \mathrm{~cm}^{-2}$ ). A cut-off is statistically required in Obs.1, but is not present in the other observations. Obs. 1 spectra were thus fitted with the highecut*powerlaw model in XSPEC. In the four first observations, an iron edge is statistically required, while no iron emission line seems to be present. In order to limit the number of fit parameters we modeled the edge with the simple edge multiplicative model. The broad band spectrum of Obs. 1 with the best fit model is represented in Fig. 2. In Obs. 4 the value of the iron edge tends to a very low value if it is left completely free to vary. We therefore allowed variations only above $6.5 \mathrm{keV}$. We note that for Obs. 5 onwards the spectrum is very likely to be contaminated by the Galactic ridge emission (Fig. 2 right panel). This manifests in particular by the appearance of an iron emission line at $\sim 6.7 \mathrm{keV}$. In order to get rid of this extra component, we modeled the ridge emission following Revnivtsev (2003), at the position of Aql X-1. The resultant spectra were then considered as extra background files for PCA and HEXTE (Fig. 2 right panel). After Obs. 5 the source is not significantly detected any more, even when the background emission of the Galaxy is modeled properly.

\subsection{Simultaneous fit of the INTEGRAL spectrum of Rev. 315 and RXTE spectra of Obs. 5}

Given the low luminosity of the source at the end of the outburst, and in order to try to obtain better constraints on the spectral parameters we fitted the INTEGRAL and RXTE spectra simultaneously. Since the observations are not strictly simultaneous, we needed to choose observations that were the closest in terms of spectral state/luminosity. Table 1 show that Rev. 315 observation is closest in time to Obs. 5 of RXTE. In addition the $20-100 \mathrm{keV}$ flux of Rev. 315 is closest to the value of the $20-100 \mathrm{keV}$ flux obtained during Obs. 5. We therefore fitted the spectrum of Rev. 315 simultaneously with those of Obs. 5. We note that No normalization constant is needed, and that a single (absorbed)

\footnotetext{
1 Conversions to $\mathrm{mCrab}$ are here made assuming a Crab-like spectrum.
} 
Table 2. Best fit parameters obtained from the fits to the INTEGRAL/ISGRI and RXTE/PCA+HEXTE spectra, with the model of an absorbed (cutoff) power law (either powerlaw or highecut* powerlaw when required). $\chi_{v}^{2}$ stands for reduced $\chi^{2}$. All errors are given at the $90 \%$ level. Upper limits are given at $90 \%$. ${ }^{\star}$ The value of the edge was constrained to be higher than $6.5 \mathrm{keV}$. The $2-20 \mathrm{keV}$ flux of the INTEGRAL observations are extrapolated.

\begin{tabular}{|c|c|c|c|c|c|c|c|c|}
\hline \multirow[t]{2}{*}{ Obs. } & \multirow[t]{2}{*}{$\begin{array}{l}\text { Edge energy } \\
\quad(\mathrm{keV})\end{array}$} & \multirow[t]{2}{*}{$\max \tau$} & \multirow[t]{2}{*}{$\Gamma$} & \multirow[t]{2}{*}{$\begin{array}{c}E_{\text {cut }} \\
(\mathrm{keV})\end{array}$} & \multirow[t]{2}{*}{$\begin{array}{l}E_{\text {fold }} \\
\mathrm{keV}\end{array}$} & \multicolumn{2}{|c|}{$\begin{array}{c}\text { Unabs. Flux } \\
\left(\times 10^{-10} \mathrm{erg} \mathrm{cm}^{-2} \mathrm{~s}^{-1}\right)\end{array}$} & \multirow[t]{2}{*}{$\begin{array}{c}\chi_{v}^{2} \\
\text { (d.o.f.) }\end{array}$} \\
\hline & & & & & & $2-20 \mathrm{keV}$ & $20-100 \mathrm{keV}$ & \\
\hline \multicolumn{9}{|c|}{ INTEGRAL } \\
\hline Rev. 305 & & & $1.93 \pm 0.25$ & $29_{-4}^{+5}$ & $49_{-10}^{+14}$ & 33.9 & 19.0 & 0.98 (19) \\
\hline Rev. 315 & & & $2.4_{-0.5}^{+0.7}$ & & & 4.01 & 1.3 & $0.5(1)$ \\
\hline \multicolumn{9}{|c|}{ RXTE } \\
\hline Obs. 1 & $7.7 \pm 0.2$ & $0.14 \pm 0.02$ & $1.88 \pm 0.01$ & $37_{-11}^{+23}$ & $52.9_{-38}^{+45}$ & 9.6 & 6.8 & $0.83(57)$ \\
\hline Obs. 2 & $7.6 \pm 0.2$ & $0.15 \pm 0.003$ & $1.91 \pm 0.01$ & & & 8.2 & 6.8 & $0.96(59)$ \\
\hline Obs. 3 & $7.0 \pm 0.2$ & $0.14 \pm 0.003$ & $1.89 \pm 0.02$ & & & 5.3 & 4.8 & $1.29(59)$ \\
\hline Obs. 4 & $<7.1^{\star}$ & $<0.18$ & $1.93 \pm 0.03$ & & & 2.6 & 2.1 & $1.26(57)$ \\
\hline Obs. 5 & & & $1.84 \pm 0.07$ & & & 0.8 & 0.7 & $0.91(34)$ \\
\hline \multicolumn{9}{|c|}{ INTEGRAL+RXTE } \\
\hline
\end{tabular}
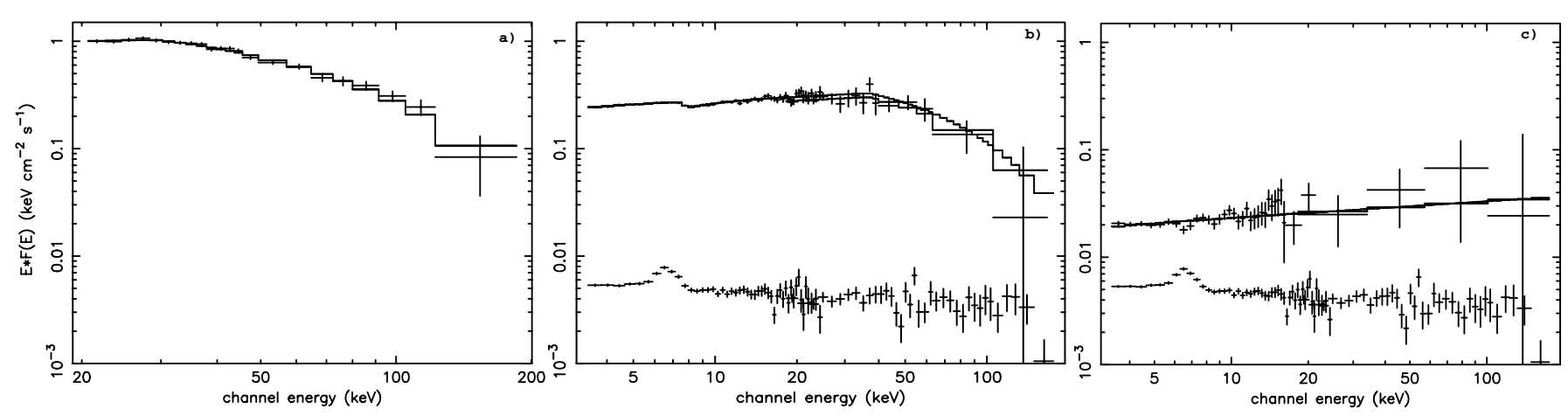

Fig. 2. a) INTEGRAL/ISGRI 20-200 keV unfolded spectrum of Aql X-1 at the maximum of the outburst, during revolution 305 . b) and c) RXTE/PCA and HEXTE spectra of Obs. 1 b), and Obs. 5 c). In panels b) and c) the lower spectra represent the emission of the Galactic ridge at the position of Aql X-1 as deduced from Revnivtsev (2003).The best fit model is superimposed on the spectrum.

power law gives a good representation of the data. The best fit parameters are reported in Table 2. The spectra from ISGRI and HEXTE are compatible, giving further confidence in the spectral parameters obtained at the peak of the outburst.

\section{Discussion}

We report the analysis of high energy INTEGRAL and RXTE observations of a faint Soft X-ray outburst of Aql X-1 $\left(\mathrm{F}_{1-12} \sim\right.$ $90 \mathrm{mCrab}$ at maximum, Fig. 1). When looking at high energies, however, the situation is quite different. At the peak of the outburst the source is clearly detected up to $\sim 150 \mathrm{keV}$ with INTEGRAL (Fig. 2). Although the lack of simultaneous data below $20 \mathrm{keV}$ renders it difficult to obtain the source photon index with high accuracy, the spectrum is typical of a hard state, with a high energy cut-off at $\sim 30 \mathrm{keV}$. Assuming this model is valid down to $1 \mathrm{keV}$, the $1-200 \mathrm{keV}$ luminosity is $\sim 2 \times 10^{37} \mathrm{erg} / \mathrm{s}$ (with $d=5 \mathrm{kpc}$ ). This is the range of luminosities at which Aql X-1 had been observed to undergo a transition to a soft state in the 1997 and 1999 outbursts (Zhang et al. 1998; Maccarone \& Coppi 2003) $\left(L=1.6-2.2 \times 10^{37} \mathrm{erg} / \mathrm{s}\right.$ at $5 \mathrm{kpc}$ in 1999), and clearly much higher than the luminosity at which it had returned to the hard state in $1999\left(\sim 2.4 \times 10^{36} \mathrm{erg} / \mathrm{s}\right.$ at $5 \mathrm{kpc}$, Maccarone \& Coppi 2003). Note that to check the consistency of the extrapolated flux, the web tool PIMMS was used to estimate the ASM flux given the ISGRI fitted spectral parameters, 20-100 keV flux and observed Nh; this returned an ASM count rate of $\sim 6 \mathrm{cts} / \mathrm{s}$, which is consistent with the observations in Fig. 1. When comparing this episode of hard state with e.g. the initial hard state episode of the 1999 outburst (Maccarone \& Coppi 2003), at similar luminosities, we detect the source up to higher energy than in 1999. Although this may be the results of the higher sensitivity of INTEGRAL/ISGRI as compared to $R X T E / H E X T E$, there are clearly physical differences between both episodes. In fact while the luminosity is close to that at which the state transition occured in 1999, the temperature of the corona (close to the folding energy of the exponential cutoff in our model, see e.g. Rodriguez et al. 2003), is higher here $(\sim 50 \mathrm{keV})$ than in 1999 ( 10 keV, Maccarone \& Coppi 2003). This higher temperature of the corona likely indicates that the accretion disk is cooler and its inner edge is further out than in 1999. A necessary condition for the transition to occur would then be related to physical parameters of the disk/corona, and not only to the total luminosity (usually taken to be equivalent to the mass accretion rate) of the source. Although based on different behaviors, similar conclusions were drawned during outbursts of XTE J1550-564 (Homan et al. 2001; Rodriguez et al. 2003).

The RXTE monitoring of the source, close to the end of the outburst, shows that the spectral parameters, in particular the photon power law index, do not evolve significantly. The decay corresponds to an overall decrease of the source luminosity, compatible with a global decrease of the accretion rate (as seen during the outburst decay of transient sources, eg XTE J1550-564 (Rodriguez et al. 2003). In fact Aql X-1and 
XTE J1550-564 share some interesting similarities: they both underwent hard state outbursts (e.g Sturner \& Shrader 2005, and the present study), they both showed hard X-ray flares prior to outbursts (Yu et al. 2003; Yu 2004), the analysis of their power spectra can show the presence of low frequency QPOs at frequencies 1-20 Hz during periods characterized by hard spectra (Yu et al. 2003; Rodriguez et al. 2004b), and they both have low period of recurence (although no real period can be claim for either of the sources). It is well known that the (normal) outbursts evolution are comparable in both the neutron star and black hole transients in many aspects (e.g. Yu et al. 2003, 2004). We show here that not only normal outbursts are comparable, but that both type of systems can undergo failed/mini or low intensity hard state outbursts, suggesting that the mechanism driving the transition is similar in both sources. During the "failed outburst" episode of XTE J1550-564, Sturner \& Shrader (2005) suggested that more than one physical mechanisms could give rise to outbursts in this source, and that the faint outburst could correspond to a discrete accretion event. This possibility could also explain the low state outburst in Aql X-1. In that case however the shape of the outburst should be FRED-like when here it has a shape closer to a triangle (Fig. 1). In the future a wider spectral coverage during those episodes of hard state outburst in neutron star and black hole systems, will help us to understand the parameter(s) triggering or not normal/major outburst.
Acknowledgements. The authors thank the anonymous referee for his/her fruitful comments. This work is based on observations with INTEGRAL, an ESA mission with instruments and science data centre funded by ESA member states (especially the PI countries: Denmark, France, Germany, Italy, Switzerland, Spain), Czech Republic and Poland, and with the participation of Russia and the USA. This reserach has made use of data obtained through the High Energy Astrophysics Science Archive Center Online Service, provided by the NASA/Goddard Space Flight Center.

\section{References}

Chevalier, C., Ilovaisky, S. A., Leisy, P., \& Patat, F. 1999, A\&A, 347, L51 Grebenev, S., Belanger, G., Bodaghee, A., et al. 2005, Atel, 452

Harmon, B. A., Wilson, C. A., Tavani, M., et al. 1996, A\&AS, 120, 197 Homan, J., Wijnands, R., van der Klis, M., et al. 2001, ApJS, 132, 377 Lebrun, F., Leray, J. P., Lavocat, P., et al. 2003, A\&A, 411, L141

Maccarone, T. J., \& Coppi, P. S. 2003, A\&A 399, 1151

Maccarone, T. J. 2005, MNRAS, 360, L68

Maitra, D., \& Bailyn, C. D. 2004, ApJ, 608, 444

Maitra, D., \& Bailyn, C. D. 2005a, Atel, 450

Maitra, D., \& Bailyn, C. D. 2005b, presented in A LIFE WITH STARS, a meeting in honor of Ed van den Heuvel, Amsterdam, 22-26 August 2005 [arXiv : astro-ph 0510329] Miyamoto, S., Kitamoto, S., Hayashida, K., \& Egoshi, W. 1995, ApJ, 442, L13 Revnivtsev, M. 2003, A\&A, 410, 865

Rodriguez, J., Corbel, S., \& Tomsick, J. A. 2003, ApJ, 595, 1032

Rodriguez, J., Hannikainen, D. C., \& Mowlavi, N. 2004a, Atel, 280

Rodriguez, J., Corbel, S., Kalemci, E., Tomsick, J. A., \& Tagger, M. 2004b, ApJ, 612, 1018 Rodriguez, J., \& Shaw, S. E. 2005, Atel, 660

Rodriguez, J., Bodaghee, A., Kaaret, P., et al. 2006, MNRAS, 366, 274

Rutledge, R. E., Bildsten, L., Brown, E. F., Pavlov, G. G., \& Zavlin, V. E. 2001, ApJ, 559, 1054

Sturner, S. J., \& Shrader, C. R. 2005, ApJ, 625, 923

Yu, W., Klein-Wolt, M., Fender, R., \& van der Klis, M. 2003, ApJ, 589, L33

Yu, W. 2004, NuPhS., 132, 668

Zhang, S. N., Yu, W., \& Zhang, W. 1998, ApJ, 494, L71 